\title{
Non-Gaussian stochastic gravitational waves from phase transitions
}

\author{
Soubhik Kumar, ${ }^{a, b}$ Raman Sundrum ${ }^{c}$ and Yuhsin Tsai ${ }^{d}$ \\ ${ }^{a}$ Berkeley Center for Theoretical Physics, Department of Physics, \\ University of California, Berkeley, CA 94720, U.S.A. \\ ${ }^{b}$ Theoretical Physics Group, Lawrence Berkeley National Laboratory, \\ Berkeley, CA 94720, U.S.A. \\ ${ }^{c}$ Maryland Center for Fundamental Physics, Department of Physics, \\ University of Maryland, College park, MD 20742, U.S.A. \\ ${ }^{d}$ Department of Physics, University of Notre Dame, \\ IN 46556, U.S.A. \\ E-mail: soubhik@berkeley.edu, raman@umd.edu, ytsai3@nd.edu
}

ABSTRACT: Cosmological phase transitions in the primordial universe can produce anisotropic stochastic gravitational wave backgrounds (GWB), similar to the cosmic microwave background (CMB). For adiabatic perturbations, the fluctuations in GWB follow those in the $\mathrm{CMB}$, but if primordial fluctuations carry an isocurvature component, this need no longer be true. It is shown that in non-minimal inflationary and reheating settings, primordial isocurvature can survive in GWB and exhibit significant non-Gaussianity (NG) in contrast to the CMB, while obeying current observational bounds. While probing such NG GWB is at best a marginal possibility at LISA, there is much greater scope at future proposed detectors such as DECIGO and BBO. It is even possible that the first observations of inflation-era NG could be made with gravitational wave detectors as opposed to the CMB or Large-Scale Structure surveys.

KeYwords: Cosmology of Theories beyond the SM, Beyond Standard Model

ArXiv ePrint: 2102.05665 


\section{Contents}

1 Introduction 1

2 Gravitational waves from phase transitions 3

3 Anisotropic gravitational wave sky 4

4 Non-Gaussian gravitational waves with adiabatic perturbations $\quad 6$

$\begin{array}{llll}5 & \text { Non-Gaussian gravitational waves with isocurvature perturbations } & 7\end{array}$

6 A non-Gaussian hidden sector model 11

7 Conclusions 12

A Derivations of large-scale CMB and GW anisotropy 13

$\begin{array}{lll}\text { A.1 Large-scale CMB anisotropy } & 14\end{array}$

$\begin{array}{lll}\text { A.2 } & \text { Large-scale GW anisotropy } & 14\end{array}$

\section{Introduction}

Since the first gravitational wave (GW) signal was detected in 2015 [1], LIGO and VIRGO have continuously observed new astronomical events like the merging of massive black holes and neutron stars [2] with a surprisingly high event rate. Looking forward, we can hope to see cosmological signatures at the next generation GW detectors including LISA [3], KAGRA [4], LIGO-India [5], Einstein Telescope (ET) [6], Cosmic Explorer (CE) [7], DECIGO [8, 9], TianGO [10], BBO [11, 12], NANOGrav [13], EPTA [14], PPTA [15] and SKA [16] through the observation of a stochastic GW background (GWB) [17, 18].

Such a stochastic GWB can originate, for example, if a new beyond Standard Model (BSM) physics process violently changes the energy density of the early universe through a strong first order phase transition (PT). For a review see [19]. It is possible that the associated BSM particles are too heavy or too weakly coupled to the Standard Model (SM) particles to be studied at particle colliders, in which case the GW frequency spectrum would give us unique insight into the new physics (for example, see [20-25]). Alternately, the GW signal may be accompanied by BSM collider discoveries, providing invaluable complementary views of the new physics (for example, see [26]). Unlike photons, GW from an early PT can propagate almost freely though the cosmological plasma to reach us. In this way, they can probe deep inside the primordial "dark age" before the Big-Bang Nucleosynthesis (BBN). Therefore, by studying the large-scale anisotropy in the GWB 
map, we can obtain a complementary insight into the nature of inflationary fluctuations relative to the Cosmic Microwave Background (CMB) and Large-Scale Structure (LSS).

If the PT happens at roughly $T \sim \mathrm{TeV}$, as motivated within many extensions of the $\mathrm{SM}$, the observable universe today would contain $\sim 10^{43}$ Hubble patches during the PT time. Therefore, given the limited angular resolution of GW detectors (e.g., $\delta \theta \sim \mathcal{O}(0.1)$ rad for LISA [27, 28]), the resulting GW from the PT time would form a diffuse background since the GW arriving from every direction in the sky is an average of GW originating from a very large number of Hubble volumes undergoing PT. With this large statistics of the number of causally independent Hubble patches that went through the PT, the average temperature when the PT completes in each finite region of the sky is nearly identical and equals $\sim T_{c}$, the critical temperature for the PT. However, since the thermal history of each Hubble patch depends on the primordial energy density fluctuation, each point on the $T=T_{c}$ surface has a varying distance in redshift from us. This leads to a modulation of the GW energy density $\rho_{\mathrm{GW}}(\theta, \phi)$ as a function of angles $\theta, \phi$ on the sky,

$$
\rho_{\mathrm{GW}}(\theta, \phi)=\bar{\rho}_{\mathrm{GW}}+\delta \rho_{\mathrm{GW}}(\theta, \phi),
$$

and any stochastic GWB produced in the early universe must be anisotropic. ${ }^{1}$ As discussed in [32], the GWB power spectrum $\left\langle\delta \rho_{\mathrm{GW}} \delta \rho_{\mathrm{GW}}\right\rangle$ and cross correlation with the $\mathrm{CMB}\left\langle\delta \rho_{\mathrm{GW}} \delta \rho_{\mathrm{CMB}}\right\rangle$ can probe the quantum fluctuations of the inflation-era fields that reheated the PT sector, without presuming that this is identical to the source of fluctuations in the CMB and LSS. While such GWB anisotropies would be challenging to measure, it was argued in [32] that they were within the reach of proposed detectors for plausibly strong PT.

Moreover, just as primordial non-Gaussianity (NG) in the CMB or LSS can arise as reflections of interactions of the inflationary (and possibly other) fields, and is being actively searched for, it is possible for the GWB fluctuations to also exhibit NG. This would be captured by the GWB bispectrum correlator $\left\langle\delta \rho_{\mathrm{GW}}\left(\hat{n}_{1}\right) \delta \rho_{\mathrm{GW}}\left(\hat{n}_{2}\right) \delta \rho_{\mathrm{GW}}\left(\hat{n}_{3}\right)\right\rangle$ where $\hat{n}_{i}$ 's for $i=1,2,3$ denote three angles on the sky. Again, this GWB bispectrum does not have to closely approximate that of the CMB if the PT sector and SM are originally reheated by different (combinations of) reheating fields, with different interactions.

In this work, we will study such GWB NG, and the mechanism by which they may be significantly stronger than (the bounds on) NG in the CMB and LSS, and plausibly observable at future GW detectors. After production, GWs free stream to us and hence the primordial GWB NG would not be significantly affected by gravitational clustering which, however, is important for CMB and (especially) LSS NG.

When discussing the three point function of GWB, it is shown in [33, 34] that the correlation function $\left\langle h^{3}\right\rangle$, with $h$ denoting an individual GW fluctuation, is almost impossible to measure - both due to the propagation of GW in a perturbed background that would de-correlate the phases in $h$, and the difficulty in separating $h$ with nearby frequencies in measurements with finite durations. However the bispectrum $\left\langle\delta \rho_{\mathrm{GW}}^{3}\right\rangle$ in the spatial distribution of the GW energy density does not depend on the initial phase of GW and

\footnotetext{
${ }^{1}$ For ansitropic GW signals from astrophysical sources, see e.g. [29-31].
} 
we can always measure this bispectrum after obtaining a map of $\delta \rho_{\mathrm{GW}}$ in the GWB. The prospects of GW map-making have been discussed previously in the literature, for a review see e.g. [35], along with some recent work [36, 37]. For previous discussions on anisotropies and non-Gaussianity of GWB, see [38, 39].

This paper is organised as follows. After reviewing the properties of a stochastic GWB from first order PT, we estimate to what extent the anisotropies of such a background can be visible at future GW detectors. Following this, we discuss NG of GWB both in the case of adiabatic and isocurvature perturbations. We find that for the latter case, provided the astrophysical GW "foreground" from binary mergers can be subtracted, future GW detectors would be able to probe primordial NG in a stronger way compared to the CMB and especially, LSS. We then give a simple model that demonstrates that the NG in GW can be significantly large while ensuring theoretical control and obeying observational bounds. Finally, we conclude.

\section{Gravitational waves from phase transitions}

GW from first order PT get generated due to three processes: collisions of nucleating bubble walls, magnetohydrodynamic (MHD) turbulence and sound waves in the plasma (for recent discussions see [40, 41] and references therein). According to simulations [4143], the MHD turbulence and sound waves can contribute dominantly to the generation of GW compared to bubble collisions, but those are dependent on details of the plasma and model-specifics, and are being actively researched. ${ }^{2}$ On the other hand, through the envelope approximation, the contribution of bubble-wall collisions are analytically better understood [44-48] and therefore, we focus only on this contribution. Given that we will be studying very small GWB and its correlation functions, this is a conservative approach. Of course, in the context of a given microscopic BSM dynamics responsible for the first order PT, the signals from sound waves and turbulence can dominate, however, that would only increase the GW signals considered below. We note that while the more recently considered "bulk-flow approximation" $[49,50]$ can change the frequency dependence of GW away from the peak, the peak amplitude roughly matches the one from the envelope approximation for some models, as seen in [51]. This conclusion regarding the peak amplitude remains true for the new estimates of GW obtained in $[51,52]$ as well. Hence we continue to use the result from [48] for the peak amplitude, as needed for our discussion on GW anisotropy, using the envelope approximation. This will allow us to give simple model-independent estimates/lower-bounds of the overall anisotropy and NG in order to see if they can be feasibly detected, while deferring modeling/calculations of the precise frequency spectrum as long as its peak lies within the sensitivity band of proposed detectors.

The peak energy density of GW from bubble-wall collisions in a thin-wall approximation, expressed in terms of the critical density today, is given by [48]

$$
\Omega_{\mathrm{GW}}^{\mathrm{peak}} h^{2}=1.3 \times 10^{-6}\left(\frac{H_{\mathrm{PT}}}{\beta}\right)^{2}\left(\frac{\alpha}{1+\alpha}\right)^{2} .
$$

\footnotetext{
${ }^{2}$ Their relative contributions depend also on efficiency factors that govern how much of the latent heat released during PT goes into accelerating the bubble walls, sound waves and turbulence.
} 
In the above, the parameter $\beta$ essentially captures the inverse duration of the $\mathrm{PT}$, is approximately given by $\beta / H_{\mathrm{PT}} \equiv d \ln \Gamma / d t \approx-4+T_{n} d S_{b} /\left.d T\right|_{T_{n}}$ in terms of the bubble nucleation rate $\Gamma$ and the bounce action $S_{b}$ at the nucleation temperature $T_{n}$. The quantity $\alpha=\rho_{\mathrm{vac}} / \rho_{\mathrm{rad}}$ is the ratio of the vacuum energy density released during the PT to the energy density of radiation bath. The Hubble parameter at the time of the $\mathrm{PT}$ is given by $H_{\mathrm{PT}} \approx \sqrt{(8 \pi / 3) G_{N} \rho_{\mathrm{tot}}}$, and we assume the effective number of degrees of freedom $g_{*} \sim 100$. Furthermore, we have considered the scenario in which the bubble walls move close to the speed of light, and efficiency factor $\kappa_{b} \simeq 1$, i.e. most of the latent heat of the PT is used up in accelerating the bubble walls. ${ }^{3}$ The quantity $\beta / H_{\mathrm{PT}}$ is model-dependent and can range between few $-\mathcal{O}(100)$, in models in the literature with a strong first-order PT [54-59]. The GW frequencies are redshifted and the peak frequency is given by,

$$
\omega_{\mathrm{GW}}^{\text {peak }}=0.04 \mathrm{mHz}\left(\frac{\beta}{H_{\mathrm{PT}}}\right)\left(\frac{T_{n}}{\mathrm{TeV}}\right) .
$$

\section{Anisotropic gravitational wave sky}

In the simplest scenario where there is a single, adiabatic source of primordial fluctuations, such as single-field inflation where the inflaton decays to reheat all sectors, the primordial fluctuations in each sector will be identical. This means the perturbation of the GWB, follows the almost scale-invariant adiabatic result, and is determined in terms of the primordial power spectrum $A_{s}: \delta \rho_{\mathrm{GW}}=\frac{4}{3} \sqrt{A_{s}} \rho_{\mathrm{GW}} \approx 6 \times 10^{-5} \rho_{\mathrm{GW}}[60]$, just as for the CMB. Therefore

$$
\begin{aligned}
\delta \Omega_{\mathrm{GW}}^{\text {peak }} h^{2} & =\Omega_{\mathrm{GW}}^{\text {peak }} h^{2} \times\left(\frac{\delta \rho_{\mathrm{GW}}}{\rho_{\mathrm{GW}}}\right) \\
& \approx 8 \times 10^{-11}\left(\frac{H_{\mathrm{PT}}}{\beta}\right)^{2}\left(\frac{\alpha}{1+\alpha}\right)^{2} .
\end{aligned}
$$

Here to go to the second line, we used eq. (2.1) and $\delta \rho_{\mathrm{GW}} / \rho_{\mathrm{GW}}=\frac{4}{3} \sqrt{A_{s}}$. The quantity $\delta \Omega_{\mathrm{GW}}^{\text {peak }} h^{2}$ can be thought of as the 'going rate' of very large-scale GW anisotropies and can be used to determine the smallest scale that can be probed at a given experiment, as we will see now.

In analogy to the CMB, we can calculate the two point function of GW perturbation $\delta_{\mathrm{GW}} \equiv \delta \rho_{\mathrm{GW}} / \rho_{\mathrm{GW}}$

$$
C^{\mathrm{GW}}(\theta) \equiv\left\langle\delta_{\mathrm{GW}}\left(\hat{n}_{1}\right) \delta_{\mathrm{GW}}\left(\hat{n}_{2}\right)\right\rangle,
$$

and the coefficient of Legendre polynomials $C_{\ell}^{\mathrm{GW}}$ from

$$
C^{\mathrm{GW}}(\theta)=\frac{1}{4 \pi} \sum_{\ell}(2 \ell+1) C_{\ell}^{\mathrm{GW}} P_{\ell}(\cos \theta)
$$

with $\hat{n}_{1} \cdot \hat{n}_{2}=\cos \theta$. In the above, $\delta_{\mathrm{GW}}$ would get additional imprint from the Sachs-Wolfe (SW) and the integrated Sachs-Wolfe (ISW) effects [61, 62]. Since the ISW effect is a

\footnotetext{
${ }^{3}$ More precisely, $\kappa_{b}=1-\frac{\alpha_{\infty}}{\alpha}$ where $\alpha_{\infty}$ is a threshold value such that if $\alpha>\alpha_{\infty}$, then wall velocity $v_{w} \approx 1$. The precise value of $\alpha_{\infty}$ is model-dependent but can be $\sim 0.01$ [40,53]. With a choice of $\alpha \gtrsim 0.01$, we then get $\kappa_{b} \simeq 1$.
} 
known, standard $\Lambda \mathrm{CDM}$ contribution in our setup, we only consider the SW effect, and take $\delta_{\mathrm{GW}}$ as the sum of the primordial density contrast $\delta_{\mathrm{GW}}^{\text {prim }}$ and Newtonian potential $\Phi$, $\delta_{\mathrm{GW}}=\delta_{\mathrm{GW}}^{\text {prim }}+4 \Phi$. Here we are using the Newtonian gauge,

$$
d s^{2}=a^{2}(\eta)\left(-(1+2 \Phi) d \eta^{2}+(1-2 \Psi) d \vec{x}^{2}\right),
$$

to characterize metric fluctuations, with $a(\eta)$ being the scale factor written in terms of the conformal time $\eta$ and $\Phi, \Psi$ are Newtonian gauge potentials. More detailed discussions on the characterization of GW anisotropy can be found in e.g. [30, 63-66]. From the CMB observation, besides small deviations coming from the physics of inflation/reheating [67], we expect the large-scale GWB perturbation should be almost scale-invariant. An exact scale invariant spectrum would correspond to

$$
C_{\ell}^{\mathrm{GW}} \propto[\ell(\ell+1)]^{-1} .
$$

Given eq. (3.2) for large-scale anisotropies and the falloff for large- $\ell$ (small-scale anisotropies) in eq. (3.6) we see that the anisotropic signal would fall below detector sensitivity at an $\ell_{\max } \simeq\left(\delta \Omega_{\mathrm{GW}}^{\text {peak }} h^{2}\right) /\left(\Omega_{\mathrm{GW}}^{\mathrm{det}} h^{2}\right)$. Here $\Omega_{\mathrm{GW}}^{\mathrm{det}} h^{2}$ the GW sensitivity for a fixed choice of Signal-to-Noise ratio (SNR). This assumes that the angular resolution of the detector is sufficient to measure such $\ell_{\max }$ modes. If not, then the maximum observable $\ell$ would be given just by the angular resolution.

Our ability to observe such GW anisotropies will be limited by detector sensitivity, as well as our understanding and ability to subtract the GW "foregrounds" arising from astrophysical mergers. The first of these limitations can be estimated simply as follows. The best LISA sensitivity, with SNR $>1$, is $\Omega_{\mathrm{GW}}^{\mathrm{LISA}} h^{2} \gtrsim 2 \times 10^{-14}$ in the frequency range $\omega_{\mathrm{GW}}^{\text {peak }} \approx 1-10 \mathrm{mHz}$, see e.g. [53, 68]. If $\left(\beta / H_{\mathrm{PT}}\right)^{2}=10$ and $T_{P T} \approx 10-100 \mathrm{TeV}$, the GW frequency is inside this range, and with $\alpha \sim \mathcal{O}(0.1), \delta \Omega_{\mathrm{GW}} h^{2} \sim 10^{-13}$ from eq. (3.2). Given the discussion below eq. (3.6), we see that for such values $\alpha, \beta$, LISA sensitivity would be able to probe $\ell_{\max } \lesssim 10$ which is within its expected angular resolution (see [28,37] for discussions on LISA sensitivity to stochastic GWB). Still it is very challenging to probe beyond $\ell \approx \mathcal{O}(10)$ in LISA. This is where proposed space-based missions such as DECIGO $[8,9]$ and BBO $[11,12]$ can make significant progress. The best DECIGO and $\mathrm{BBO}$ sensitivity, with $\mathrm{SNR}>1$, is around $\Omega_{\mathrm{GW}}^{\mathrm{DECIGO}} h^{2} \gtrsim 10^{-17}$ (see e.g. [68, 69]), around a frequency $\omega_{\mathrm{GW}}^{\text {peak }} \approx 100 \mathrm{mHz}$ and $T_{n} \sim 10^{4} \mathrm{TeV}$. Given the discussion below eq. (3.6), we can observe $\delta \Omega_{\mathrm{GW}} h^{2}$ up to $\ell \approx 100$ in DECIGO and BBO even for a shorter PT $\left(\beta / H_{\mathrm{PT}}\right)^{2}=100$ with $\alpha \sim \mathcal{O}(0.1)$. Such an $\ell_{\max } \approx 100$ is within the DECIGO/BBO angular resolution. For a recent discussion on angular resolution of future GW detectors for various configurations, including those in deci-Hz range, see [70]. The proposed ET and $\mathrm{CE}$ detectors would probe higher frequencies $\left(\sim 1-10^{3} \mathrm{~Hz}\right)$ than LISA and be sensitive to GW background anisotropies [71]. However, with the lower sensitivity of ET compared to LISA it would be challenging to see NG unless $\left(\beta / H_{\mathrm{PT}}\right)^{2} \sim$ few which is hard to realize in models. On the other hand, CE can perform similarly to LISA.

But of course, to achieve the above targets we must be able to identify and "subtract" the astrophysical GW foregrounds that can be competitive to the cosmological GW 
anisotropies in the relevant frequency ranges. These can take the form of unresolved or resolvable merger events, such as those involving white dwarfs, neutron stars and black holes (see [72] for a review). Our ability to extract primordial GWB in the presence of such astrophysical foregrounds has been studied in several papers including [73-81]. In particular, refs. [73, 74] focus on the subtraction problem for BBO/DECIGO frequencies, and shows that almost all the mergers can be individually subtracted to isolate the cosmological signal [29]. However, all these studies focused on situations which the astrophysical foreground competes with the isotropic cosmological signal. Here, we are considering the possibility that the isotropic cosmological signal is very strong, much larger than the astrophysical foreground, but where the cosmological anisotropies are smaller. Nevertheless, astrophysical foreground subtraction may still be possible in this novel regime, exploiting the cosmological frequency spectrum that can be precisely measured from the large isotropic component. This deserves dedicated study, but we will proceed assuming such subtraction is possible.

\section{Non-Gaussian gravitational waves with adiabatic perturbations}

Before moving on to the GW NG, we first parametrize the CMB NG. Instead of using the conventional harmonic coefficients $a_{l m}$ of the CMB anisotropies, we describe the CMB NG by simply using the CMB density perturbations in momentum space (including the SW gravitational redshift), $\delta_{\gamma}=\delta_{\gamma}^{\text {prim }}+4 \Phi$. This will be sufficient to illustrate the essential physical effects of adiabatic and isocurvature perturbations on CMB and GW NG. Thus we parametrize the CMB bispectrum as, ${ }^{4}$

$$
F_{\mathrm{CMB}}\left(\vec{k}_{1}, \vec{k}_{2}, \vec{k}_{3}\right) \equiv \frac{2}{3} \frac{\left\langle\delta_{\gamma}\left(\vec{k}_{1}\right) \delta_{\gamma}\left(\vec{k}_{2}\right) \delta_{\gamma}\left(\vec{k}_{3}\right)\right\rangle^{\prime}}{P_{\gamma}\left(k_{1}\right) P_{\gamma}\left(k_{2}\right)+P_{\gamma}\left(k_{1}\right) P_{\gamma}\left(k_{3}\right)+P_{\gamma}\left(k_{2}\right) P_{\gamma}\left(k_{3}\right)},
$$

where $P_{\gamma}(k)=\left\langle\delta_{\gamma}(\vec{k}) \delta_{\gamma}(-\vec{k})\right\rangle^{\prime}$ is the CMB power spectrum and 's on correlators denote the fact that momentum-conserving factors of $(2 \pi)^{3} \delta\left(\vec{k}_{1}+\cdots+\vec{k}_{n}\right)$ are stripped off. The prefactor $2 / 3$ ensures that for the adiabatic perturbations, the standard definition of primordial $\mathrm{NG}[82]$ is reproduced. In the above example of single-source reheating, $\delta_{\mathrm{GW}}$ follows the same adiabatic perturbation as $\delta_{\gamma}$, and the bi-spectrum from the GW observation is

$$
\begin{aligned}
F_{\mathrm{GW}}\left(\vec{k}_{1}, \vec{k}_{2}, \vec{k}_{3}\right) & =\frac{10}{9} \frac{\left\langle\delta_{\mathrm{GW}}\left(\vec{k}_{1}\right) \delta_{\mathrm{GW}}\left(\vec{k}_{2}\right) \delta_{\mathrm{GW}}\left(\vec{k}_{3}\right)\right\rangle^{\prime}}{P_{\mathrm{GW}}\left(k_{1}\right) P_{\mathrm{GW}}\left(k_{2}\right)+P_{\mathrm{GW}}\left(k_{1}\right) P_{\mathrm{GW}}\left(k_{3}\right)+P_{\mathrm{GW}}\left(k_{2}\right) P_{\mathrm{GW}}\left(k_{3}\right)} \\
& =F_{\mathrm{CMB}}\left(\vec{k}_{1}, \vec{k}_{2}, \vec{k}_{3}\right),
\end{aligned}
$$

where the factor of $10 / 9$ ensures equality with $F_{\mathrm{CMB}}$ for adiabatic perturbations. To obtain an estimate of the observational sensitivity of future GW detectors, we first note that given an experiment, the cosmic-variance-limited precision of bispectrum measurement is determined by the number of independent modes the experiment can observe. Hence,

\footnotetext{
${ }^{4}$ also conventionally denoted as the $f_{\mathrm{NL}}$ parameter
} 
a crude cosmic variance limited sensitivity $\Delta F_{\mathrm{GW}}$ from the GWB observation which can access multipoles up to $\ell_{\max }$, is (see e.g. [83])

$$
\Delta F_{\mathrm{GW}} \sim \frac{1}{\delta_{\mathrm{GW}}} \times \frac{1}{\sqrt{\ell_{\max }\left(\ell_{\max }+1\right)}} \sim 10^{4} \ell_{\max }^{-1} .
$$

Recalling that the search of the CMB bi-spectrum has set an upper bound $\left|F_{\mathrm{CMB}}\right| \lesssim$ 5 - 50 [82] depending on the shape of NG, eq. (4.3) implies that even if we can measure the anisotropy $\delta \rho_{\mathrm{GW}}$, the bounds on $\mathrm{NG}$ from GWB will be rather weak compared to that from $\mathrm{CMB}$ unless $\ell_{\max } \gtrsim 10^{3}$. However, probing scales such small angular scales corresponding to $\ell_{\max } \gtrsim 10^{3}$ can be quite difficult even considering DECIGO and BBO, given the previously discussed benchmark points.

\section{Non-Gaussian gravitational waves with isocurvature perturbations}

The above conclusions can significantly change once we consider the universe to go through three different stages of reheating, as put forward in [32]. The inflaton $\phi$, whose fluctuations are mostly Gaussian, decays into a Hidden Sector (HS) of particles which is distinct from the BSM sector undergoing the PT, and thermally decoupled from it. We assume that the BSM sector (which includes dark matter (DM)) is reheated by a separate field $\sigma$ which is a spectator field to the inflationary dynamics. We take both the inflationary expansion and primordial perturbations to be dominated by the inflaton $\phi$, and the $\sigma$ to contribute to isocurvature perturbations. This will enable us to have significantly NG $\sigma$-fluctuations while ensuring the observational bounds are satisfied.

After the PT, the HS also decays into the BSM sector and thermalizes with it. In this way, even though both reheating processes ultimately populate the BSM sector, if $\sigma$ reheats the universe to well above the PT temperature, and the HS decay happens at a temperature below the PT, GW anisotropies will receive contributions from the fluctuations of $\sigma$. Since GWs freely stream after getting generated, the reheating mediated by the HS decays does not affect the NG nature of the GW signal. In particular, any NG of $\sigma$-fluctuations will get imprinted and preserved in NG of GW. We illustrate the history of the two reheating processes in figure 1.

We label the energy densities in the two sectors (prior to HS decay) as $\rho_{\mathrm{BSM}}$ and $\rho_{\mathrm{HS}}$, with the associated energy fractions,

$$
f_{i}=\frac{\rho_{i}}{\rho_{\mathrm{BSM}}+\rho_{\mathrm{HS}}}, \quad i=\mathrm{BSM}, \mathrm{HS} .
$$

We take $f_{\mathrm{BSM}} \ll f_{\mathrm{HS}}$ to enforce that the NG BSM sector contains a subdominant energy fraction.

To compute the NG in the present scenario we will use the gauge invariant comoving curvature perturbation on uniform-density hypersurfaces $\zeta$, to characterize cosmological perturbations [84]. In terms of the Newtonian gauge quantities eq. (3.5), $\zeta$ is given by,

$$
\zeta=-\Psi-H \frac{\delta \rho}{\dot{\rho}}
$$




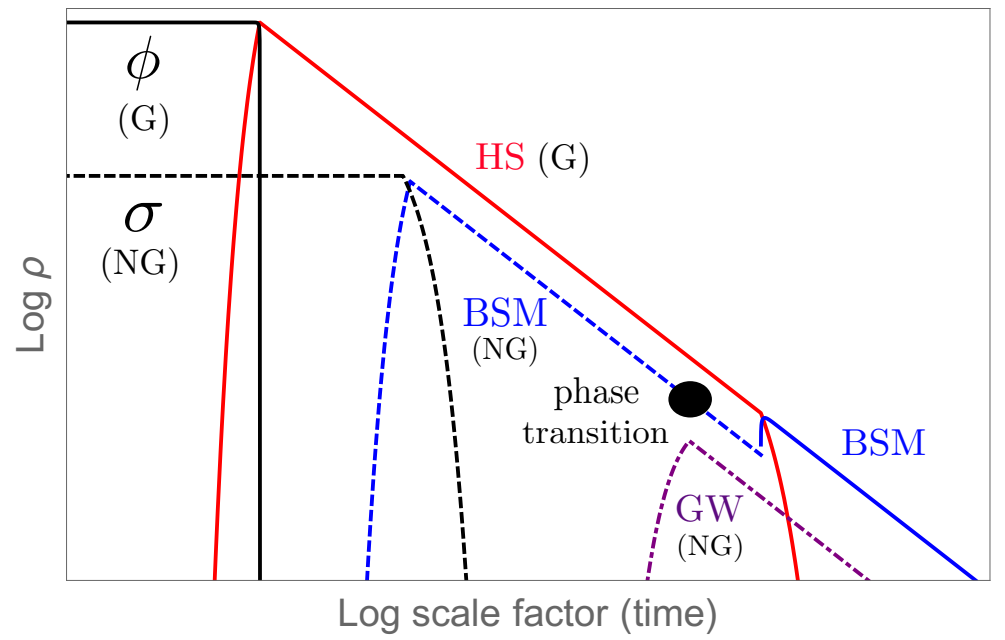

Figure 1. Cosmological history of the CMB and GWB production, along with their fluctuations. The inflaton field (solid black) carries a Gaussian $(\mathrm{G})$ fluctuation and reheats a hidden sector (HS) (red). A separate field $\sigma$ (dashed black) carries a non-Gaussian (NG) fluctuation and reheats the BSM sector (blue) where the PT occurs. Following the PT, the HS decay into the BSM sector which eventually gives rise to the CMB. Depending on the NG of $\sigma$ and the size of $f_{\mathrm{BSM}}$, the fractional energy density of the BSM sector, the GW signal (purple) can have a large NG while CMB is almost Gaussian. The plot is adapted and modified from [32].

where $\rho$ is the total energy density and the dot denotes a derivative with respect to physical time $t$. Furthermore, we define isocurvature perturbation of GW with respect to photons $\gamma$ in analogy with neutrino isocurvature perturbation, as

$$
S_{\mathrm{GW}} \equiv 3\left(\zeta_{\mathrm{GW}}-\zeta_{\gamma}\right),
$$

where the total photon perturbation is

$$
\zeta_{\gamma} \equiv f_{\mathrm{BSM}} \zeta_{\gamma_{\mathrm{BSM}}}+f_{\mathrm{HS}} \zeta_{\gamma_{\mathrm{HS}}}
$$

In the above, we have denoted $\gamma_{\mathrm{HS}(\mathrm{BSM})}$ to be the photons coming from the HS (BSM) respectively, and

$$
\zeta_{i}=-\Psi-H \frac{\delta \rho_{i}}{\dot{\rho}_{i}}
$$

denotes the gauge invariant fluctuation of a single fluid $i$. Since both $\sigma$ and $\phi$ ultimately reheat the BSM sector at high temperature where the SM and DM components are assumed to be still in equilibrium, there is no baryon, DM, or neutrino isocurvature perturbations from the reheating scenario. However, since GW are decoupled from the rest of the fluids after their production, but before HS decay, it does carry isocurvature NG from the difference between the $\delta \sigma$ and $\delta \phi$ perturbations.

We assume the universe was radiation dominated (RD) when the PT takes place. After getting generated, GW climb out of the gravitational potential well created by fluctuations 
in $\phi$ and propagate to us. Thus, large-scale GW energy density fluctuations can be written in terms of primordial perturbations as (for the derivation, see the appendix A),

$$
\delta_{\mathrm{GW}}=\delta_{\mathrm{GW}}^{\mathrm{prim}}+4 \Phi_{\mathrm{RD}}=-\frac{4}{3} \zeta_{\mathrm{RD}}+\frac{4}{3}\left(1-f_{\mathrm{GW}}\right) S_{\mathrm{GW}},
$$

where $\Phi_{\mathrm{RD}}, \zeta_{\mathrm{RD}}$ are respectively the Newtonian potential and the primordial adiabatic curvature perturbation during RD. For later convenience, we rewrite the above in terms of fluctuations of $\phi, \zeta_{\phi}$ and of $\sigma, \zeta_{\sigma}$,

$$
\delta_{\mathrm{GW}}=-\frac{4}{3} \zeta_{\phi}+\frac{4}{3} S_{\sigma}\left(1-\frac{4}{3} f_{\mathrm{BSM}}\right)
$$

where the isocurvature fluctuations of $\sigma$ is defined as, $S_{\sigma} \equiv 3\left(\zeta_{\sigma}-\zeta_{\phi}\right)$.

On the other hand, large-scale CMB energy density fluctuations are given by (for a derivation see the appendix A),

$$
\delta_{\gamma}=\delta_{\gamma}^{\text {prim }}+4 \Phi_{\mathrm{MD}}=-\frac{4}{5} \zeta_{\mathrm{RD}}+\frac{4}{15} f_{\mathrm{GW}} S_{\mathrm{GW}}
$$

where we have approximated the Universe to be almost matter dominated (MD) during CMB decoupling. This in terms of the field fluctuations read as,

$$
\delta_{\gamma}=-\frac{4}{5} \zeta_{\phi}-\frac{4}{15} f_{\mathrm{BSM}} S_{\sigma}
$$

Since the $\Delta N_{\text {eff }} \lesssim 0.4(2 \sigma)$ constraint on the effective number of new relativistic degrees of freedom inferred from the CMB and Big-bang nucleosynthesis (BBN) analyses [60, 85] requires

$$
\rho_{\mathrm{GW}} \lesssim 0.1 \rho_{\gamma}
$$

this implies, together with eq. (2.1) with $\alpha \approx f_{\mathrm{BSM}}$, a weak upper bound on $f_{\mathrm{BSM}} \lesssim 1$.

Using eqs. (5.7) and (5.9), we can now estimate the large-scale NG in CMB and GW by using eqs. (4.1) and (4.2) respectively. To satisfy the NG bounds measured using CMB, from now on we will assume inflaton perturbations are mostly Gaussian. The CMB NG is determined by eqs. (4.1) and (5.9) and in the limit $\zeta_{\phi} \gtrsim f_{\mathrm{VS}} S_{\sigma}$ they imply,

$$
F_{\mathrm{CMB}} \approx-\frac{5}{6}\left(\frac{f_{\mathrm{BSM}}}{3}\right)^{3}\left(\frac{P_{S}\left(k_{1}\right) P_{S}\left(k_{2}\right)+\text { perms. }}{P_{\phi}\left(k_{1}\right) P_{\phi}\left(k_{2}\right)+\text { perms. }}\right) F_{S_{\sigma}},
$$

where $P_{\phi}(k)=\left\langle\zeta_{\phi}(\vec{k}) \zeta_{\phi}(-\vec{k})\right\rangle^{\prime}$ and $P_{S}(k)=\left\langle S_{\sigma}(\vec{k}) S_{\sigma}(-\vec{k})\right\rangle^{\prime}$ are the inflaton and curvaton power spectrum respectively, and "perms." include the permutations $\left(k_{1}, k_{2}\right) \rightarrow\left(k_{1}, k_{3}\right)$ and $\left(k_{1}, k_{2}\right) \rightarrow\left(k_{2}, k_{3}\right)$. The quantity $F_{S_{\sigma}}=\left\langle S_{\sigma}^{3}\right\rangle /\left(\left\langle S_{\sigma}^{2}\right\rangle^{2}+\right.$ perms. $)$, schematically defined in analogy with eq. (4.1) with $S_{\sigma}$ replacing $\delta_{\gamma}$ (but without the prefactor), characterizes isocurvature NG. We see that even if isocurvature perturbations are highly non-Gaussian with $F_{S_{\sigma}} \sim 1 / S_{\sigma} \sim 10^{4}$, for $f_{\mathrm{BSM}} \lesssim 0.1$ consistent with the $\Delta N_{\text {eff }}$ constraint eq. (5.10), and $P_{S} \sim P_{\phi}$, we get $F_{\mathrm{CMB}} \lesssim 10$, satisfying the NG bounds on CMB. 


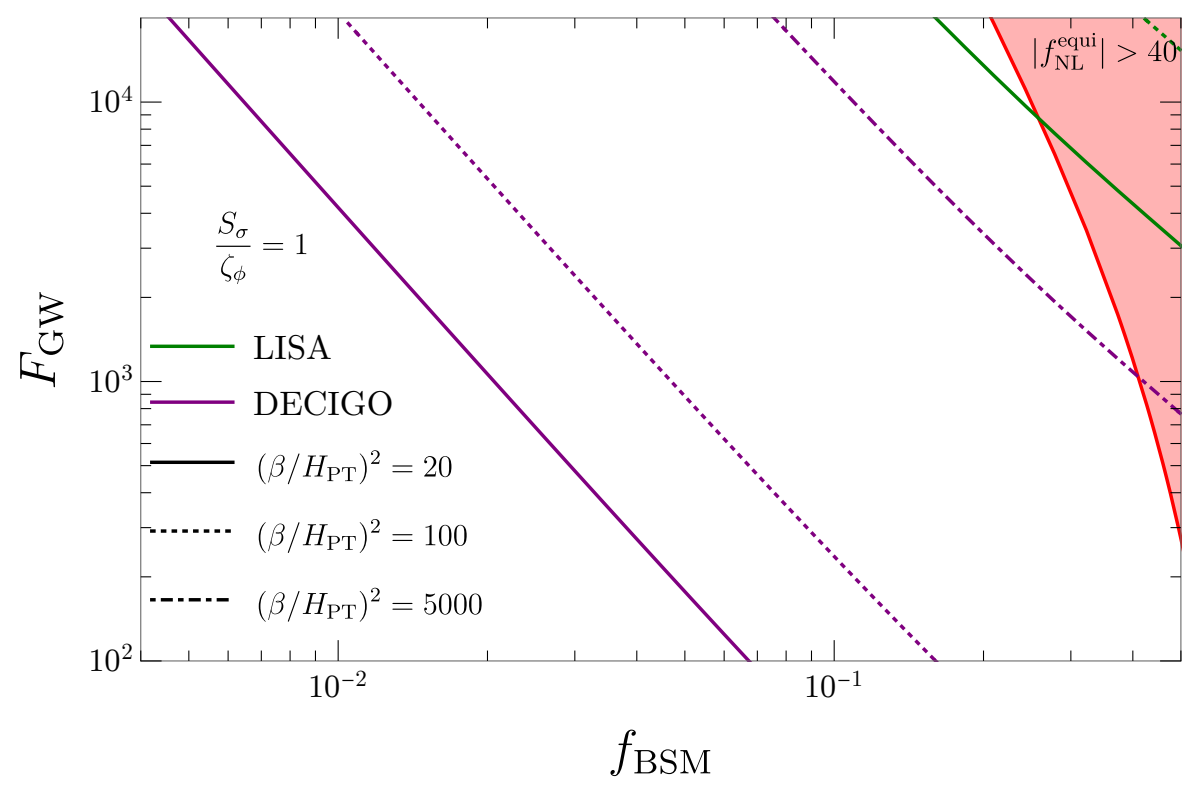

Figure 2. The projected sensitivity of the Gravitational Wave Background NG, $F_{\mathrm{GW}}$ as a function of energy fraction $f_{\mathrm{BSM}}$ of the BSM sector undergoing PT for the case of equilateral NG. In our context the PT parameter $\alpha=f_{\mathrm{BSM}}$. Depending on different assumptions of the PT parameter $\left(\beta / H_{\mathrm{PT}}\right)$, LISA and DECIGO would be able to probe regions above the green and purple lines respectively. The reach of $\mathrm{BBO}$, which we do not show explicitly for clarity, is expected to be slightly better than that of DECIGO. The region labeled $\left|f_{\mathrm{NL}}^{\text {equi }}\right|>40$ is ruled out based on the constraints on equilateral NG from Planck [82].

However, this $f_{\mathrm{BSM}}^{3}$ suppression does not appear in GW NG. In particular, eqs. (4.2) and (5.6) imply for small $f_{\mathrm{BSM}}$,

$$
F_{\mathrm{GW}}=\frac{5\left(P_{S}\left(k_{1}\right) P_{S}\left(k_{2}\right)+\text { perms. }\right) F_{S_{\sigma}}}{6\left(P_{\phi}\left(k_{1}\right)+P_{S}\left(k_{1}\right)\right)\left(P_{\phi}\left(k_{2}\right)+P_{S}\left(k_{2}\right)\right)+\text { perms }},
$$

For a simple benchmark in which the two sectors have comparable primordial power spectra, $P_{S} \sim P_{\phi}$, we see $F_{\mathrm{GW}} \sim F_{S_{\sigma}} \sim 10^{4}$ then satisfies all the observational constraints.

As discussed in eq. (4.3), $F_{\mathrm{GW}} \sim 10^{3}-10^{4}$ can be detected with $\ell_{\max } \sim \mathcal{O}(10)$. Such values of $\ell_{\max }$, can be accessed in LISA for the benchmark point discussed earlier (again assuming suitable astrophysical foreground subtraction is performed). Hence, although crude, LISA itself might give us one of the first bounds, or a detection, of non-Gaussianity from GW. As discussed above, with DECIGO and BBO, one can access $\ell_{\max } \lesssim \mathcal{O}(100)$. Correspondingly one can get to $F_{\mathrm{GW}} \sim \mathcal{O}(100)$. Note, probing such a level of HS NG using CMB or LSS requires a $F_{\mathrm{CMB}} \sim 10^{-1}$ for $f_{\mathrm{BSM}} \sim 0.1$ - challenging for LSS (given gravitationally induced non-linearities) and impossible for $\mathrm{CMB}$ for the equilateral shape.

Moving away from the above benchmark parameter choices, in figure 2 we show projections of the LISA and DECIGO sensitivities on the $F_{\mathrm{GW}}$ measurements. We focus on the case of equilateral NG $[86,87]$ for which GW can probe primordial NG in a powerful way, complementary to the CMB and LSS measurements. The limit of the $F_{\mathrm{GW}}$ measurement 
depends on the strength of the PT, the energy ratio $f_{\mathrm{BSM}}$, and the isocurvature perturbation $S_{\sigma}$. The NG signal is visible when both $\delta \Omega_{\mathrm{GW}}$ is higher than the detector sensitivity and $F_{\mathrm{GW}} \gtrsim \Delta F_{G W}$ in eq. (4.3), given good enough angular resolution. The larger $f_{\mathrm{BSM}}$ region is excluded by a comparison of eqs. (3.1) and (5.11) to the current bounds on $F_{\mathrm{CMB}}$ from the Planck (red) measurements. For equilateral NG, future LSS constraints from galaxy clustering (see e.g. [88]) are expected to be similar to the Planck constraints, and we do not show it explicitly. The $\Delta N_{\text {eff }}$ constraints from the future CMB-S4 measurement [89] are too weak to be visible in figure 2. As we can see, a large non-Gaussian signal can exist in the GWB even with the current constraint, and the corresponding $\delta \rho_{\mathrm{GW}}$ can be within the reach of DECIGO and BBO. When the PT is strong source of GW, say with $\left(\beta / H_{\mathrm{PT}}\right)^{2} \sim 20,{ }^{5}$ it might even be possible for LISA to probe an interesting and novel part of the parameter space. Lastly, in figure 2 we have focused on the case $S_{\sigma}=\zeta_{\phi}$ just for simplicity, and we have checked that similar conclusions are obtained for other choices of $S_{\sigma}$.

\section{A non-Gaussian hidden sector model}

We now discuss a simple HS model that can give rise to large NG in GW. We will assume that the spectator scalar field $\sigma$ is light during inflation so that it experiences significant quantum fluctuations, $m_{\sigma}^{2} \ll H_{\text {inf }}^{2}$, where $H_{\text {inf }}$ is the Hubble scale during inflation. We also assume the energy density in $\sigma$ both during and after inflation is subdominant. After the end of inflation, $\sigma$ dilutes like matter and eventually reheats into the BSM sector that later undergoes the PT. The isocurvature component of the BSM particles, before the HS decay into BSM, is then given by (see e.g. [90-92]),

$$
S_{\sigma}=\frac{2 \delta \sigma}{\sigma_{0}}
$$

where $\sigma_{0}$ is the originally misaligned homogeneous VEV of the $\sigma$ field and $\delta \sigma$ denotes its fluctuation. For $S_{\sigma} \sim \zeta_{\phi}$ we need, $H_{\mathrm{inf}} / \sigma_{0} \sim 10^{-4}$. Around a temperature $T \sim \mathrm{TeV}$, the BSM sector undergoes a strong first-order PT and release GWs. At the end of the PT, the BSM sector consists of the remnant BSM plasma and the generated GW, and the only species eventually contributing to $\Delta N_{\text {eff }}$ is the GW.

In the above set-up, any interaction involving $\sigma$ will induce NG in $\delta \sigma$, and eventually in GW. To characterize interactions of $\sigma$, while ensuring the radiative stability of its low mass, we will only consider a shift-symmetric derivative interaction,

$$
\mathcal{L}_{\sigma}=-\frac{1}{2}\left(\partial_{\mu} \sigma\right)^{2}-\frac{1}{2} m_{\sigma}^{2} \sigma^{2}+\frac{1}{\Lambda_{\sigma}^{4}}\left(\partial_{\mu} \sigma\right)^{2}\left(\partial_{\nu} \sigma\right)^{2}+\cdots .
$$

\footnotetext{
${ }^{5}$ In some models of $\mathrm{PT}$, modest values of $\beta / H_{\mathrm{PT}} \sim$ few are associated with supercooling and large $\alpha$ as opposed to $f_{\mathrm{BSM}}=\alpha \lesssim 0.1$ considered here. However, in our set up $\alpha \lesssim 0.1$ can arise quite easily since we consider $\mathrm{PT}$ in a sector reheated by the curvaton $\sigma$. At the end of inflation, one can have a small energy density in $\sigma$ as in figure 1. Then if the Universe undergoes supercooling, the ambient radiation energy density dilutes, and $\alpha$ increases till the time of bubble nucleation. But given a sufficiently small initial value of $\alpha_{\text {in }}$, the final $\alpha$ relevant for the PT can still be $\lesssim 0.1$ and populate the parameter space considered in figure 2 .
} 
At the classical level, we can get the equation of motion for the homogeneous background $\sigma_{0}$, which has a slow-roll approximation [86], $\ddot{\sigma}_{0} \ll H \dot{\sigma}_{0}$, under which

$$
\dot{\sigma}_{0}\left(1+4 \frac{\dot{\sigma}_{0}^{2}}{\Lambda_{\sigma}^{4}}\right) \approx-\frac{m_{\sigma}^{2}}{3 H_{\mathrm{inf}}} \sigma_{0} .
$$

Hence to only have perturbative effects from the dimension-8 operator in eq. (6.2), we will require $\dot{\sigma}_{0}^{2} / \Lambda_{\sigma}^{4} \lesssim 0.1{ }^{6}$ Importantly, the Lagrangian in eq. (6.2) also contains a non-trivial cubic interaction term

$$
\mathcal{L}_{\sigma} \supset 4 \frac{\dot{\sigma}_{0}}{\Lambda_{\sigma}^{4}} \dot{\delta \sigma}(\partial \delta \sigma)^{2}
$$

given which, the three-point function of $\delta \sigma$ can be calculated in the equilateral limit with $k_{1}=k_{2}=k_{3}=k$ as $[86]$,

$$
\left\langle\delta \sigma\left(\vec{k}_{1}\right) \delta \sigma\left(\vec{k}_{2}\right) \delta \sigma\left(\vec{k}_{3}\right)\right\rangle^{\prime}=-\frac{7}{3} \frac{\dot{\sigma}_{0}}{\Lambda_{\sigma}^{4}} \frac{H_{\mathrm{inf}}^{5}}{k^{6}} .
$$

Using this, eq. (6.1) and the definition of $F_{S_{\sigma}}$ given below eq. (5.11) we can evaluate in the equilateral limit,

$$
\left|F_{S_{\sigma}}\right|=\frac{14}{9} \frac{H_{\mathrm{inf}} \sigma_{0} \dot{\sigma}_{0}}{\Lambda_{\sigma}^{4}} .
$$

Therefore with the benchmark choice $\dot{\sigma}_{0}^{2} / \Lambda_{\sigma}^{4} \lesssim 0.1, \dot{\sigma}_{0} \sim H_{\text {inf }}^{2}, \sigma_{0} / H_{\text {inf }} \sim 10^{4}$, we can have $F_{\mathrm{GW}} \sim F_{S_{\sigma}} \lesssim$ few $\times 10^{3}$ which can be probed at LISA, and with much better precision at DECIGO and BBO.

\section{Conclusions}

We have shown that large isocurvature non-Gaussianity (NG) can be hidden inside a stochastic GW background arising from a PT, and yet be invisible even to the near-future LSS or CMB measurements. If inflation and reheating involve multiple scalar fields, the reheating from some of the scalar fields can pick up large NG perturbations and produce NG GW background even when the rest of the cosmological fluids inherit predominantly Gaussian fluctuations. In this case, future surveys of the stochastic GW signal by LISA, DECIGO, and BBO may give us the first glimpse of primordial NG quantum fluctuations in the early universe!

\section{Acknowledgments}

We are grateful to Michael Geller, Anson Hook, Jonathan Kozaczuk, Arianna Renzini, Joseph Romano and especially Neil Cornish for very useful discussions. This research was supported in part by the US-Israeli BSF Grant 2018236 and by the Maryland Center for Fundamental Physics. SK was also supported in part by the NSF grants PHY-1915314 and the U.S. DOE Contract DE-AC02-05CH11231. YT was also supported in part by the NSF grant PHY-2014165.

\footnotetext{
${ }^{6}$ Note, a choice of $\dot{\sigma}_{0}^{2} / \Lambda_{\sigma}^{4} \sim 0.1$ requires $H \lesssim \Lambda_{\sigma} \ll \sigma_{0}$. Controlled effective field theory examples of such scenarios can be constructed using bi-axion alignment models [93], as explored in some detail in [94].
} 


\section{A Derivations of large-scale CMB and GW anisotropy}

In this appendix we give a derivation of the large-scale anisotropy of CMB and GW in the context of the reheating scenario described in the main text. To this end, we denote the homogeneous metric using conformal time $\eta$ as,

$$
d s^{2}=a^{2}(\eta)\left(-d \eta^{2}+d \vec{x}^{2}\right)
$$

and the scalar metric fluctuations as [84],

$$
\delta g_{00}=-2 a^{2} \varphi ; \delta g_{0 i}=a^{2} \partial_{i} B ; \delta g_{i j}=2 a^{2}\left(-\psi \delta_{i j}+\partial_{i} \partial_{j} E\right)
$$

The relevant perturbed Einstein equations on superhorizon scales and in the absence of anisotropic stress are given by,

$$
\begin{gathered}
3 \mathcal{H}\left(\mathcal{H} \varphi+\psi^{\prime}\right)=-4 \pi G a^{2} \delta \rho, \\
\left(E^{\prime}-B\right)^{\prime}+2 \mathcal{H}\left(E^{\prime}-B\right)+\psi-\varphi=0 .
\end{gathered}
$$

Here's denote derivatives with respect to $\eta$. The conformal Hubble rate and the perturbed energy density are respectively given by $\mathcal{H}$ and $\delta \rho$. As introduced in the main text, the curvature perturbation on uniform density hypersurface is given by the gauge invariant expression,

$$
\zeta=-\psi-\mathcal{H} \frac{\delta \rho}{\rho^{\prime}}
$$

In the Newtonian gauge $B=E=0$, these equations simplify,

$$
\begin{aligned}
3 \mathcal{H}\left(\mathcal{H} \varphi+\psi^{\prime}\right) & =-4 \pi G a^{2} \delta \rho, \\
\psi & =\phi .
\end{aligned}
$$

Writing $\psi=\Psi$ and $\varphi=\Phi$ and using eqs. (A.5) and (A.6), $\zeta$ can be written as,

$$
\zeta=-\Psi-\frac{2}{3(1+w) H}(H \Phi+\dot{\Psi})
$$

where $w$ is the effective equation of state.

We will be interested in obtaining expressions for large-scale modes. For this purpose, it will be useful to have the following relations between $\zeta$ and $\Phi(=\Psi)$ during both Radiation Domination (RD) and Matter Domination (MD):

$$
\begin{aligned}
\zeta_{\mathrm{RD}} & =-\frac{3}{2} \Phi_{\mathrm{RD}} \\
\zeta_{\mathrm{MD}} & =-\frac{5}{3} \Phi_{\mathrm{MD}}
\end{aligned}
$$

In the above, we have used the constancy of $\Phi$ on super-horizon scales in the absence of non-adiabatic pressure perturbation. Now we apply the above results in the PT scenario described in the main text for which the PT takes place in a beyond Standard Model (BSM) sector reheated by $\sigma$. The hidden sector (HS), on the other hand, is reheated by inflaton, $\phi$. After the PT takes place and GW are released, the HS decays into BSM sector. 


\section{A.1 Large-scale CMB anisotropy}

The large-scale CMB anisotropies are given by, after accounting for the local redshift i.e., the SW effect,

$$
\left.\frac{\Delta T}{T}\right|_{\mathrm{CMB}}=\frac{1}{4} \delta_{\gamma}^{\text {prim }}+\Phi_{\mathrm{MD}}=\zeta_{\gamma}+2 \Phi_{\mathrm{MD}}
$$

Here $\zeta_{\gamma}$ is the gauge invariant photon perturbation, generally given by $\zeta_{\gamma}=-\psi+\frac{1}{4} \delta_{\gamma}$. Using eq. (A.9) this gives,

$$
\left.\frac{\Delta T}{T}\right|_{\mathrm{CMB}}=\zeta_{\gamma}-\frac{6}{5} \zeta_{\mathrm{MD}}
$$

Since in this scenario both the matter and neutrino number densities track the photon number density, the only isocurvature is in GW. Thus we have $\zeta_{\mathrm{MD}}=\zeta_{\gamma}$ implying

$$
\left.\frac{\Delta T}{T}\right|_{\mathrm{CMB}}=-\frac{1}{5} \zeta_{\gamma} .
$$

To compare with the Planck isocurvature bounds it is more convenient to write $\left.\frac{\Delta T}{T}\right|_{\text {CMB }}$ as a linear combination of curvature and isocurvature perturbations. Using the fact that after HS decay, we only have photons, neutrinos $(\nu)$ and GW diluting as radiation,

$$
\begin{aligned}
\zeta_{\mathrm{RD}} & =\left(1-f_{\nu}-f_{\mathrm{GW}}\right) \zeta_{\gamma}+f_{\nu} \zeta_{\nu}+f_{\mathrm{GW}} \zeta_{\mathrm{GW}} \\
& =\zeta_{\gamma}+\frac{1}{3} f_{\mathrm{GW}} S_{\mathrm{GW}},
\end{aligned}
$$

we get

$$
\left.\frac{\Delta T}{T}\right|_{\mathrm{CMB}}=-\frac{1}{5} \zeta_{\mathrm{RD}}+\frac{1}{15} f_{\mathrm{GW}} S_{\mathrm{GW}} .
$$

In the above we have used the energy density fractions, $f_{i}=\rho_{i} / \rho_{\text {tot }}$ for the three species $i=\gamma, \nu, \mathrm{GW}$ with $\rho_{\text {tot }}$ being the total energy density during RD. To compute NG, on the other hand, it is more convenient if we express $\left.\frac{\Delta T}{T}\right|_{\mathrm{CMB}}$ in terms of uncorrelated inflationary perturbations. For this purpose we write,

$$
\begin{aligned}
\left.\delta_{\gamma} \equiv 4 \frac{\Delta T}{T}\right|_{\mathrm{CMB}} & =-\frac{4}{5}\left(\zeta_{\gamma_{\mathrm{HS}}}+f_{\mathrm{BSM}}\left(\zeta_{\gamma_{\mathrm{BSM}}}-\zeta_{\gamma_{\mathrm{HS}}}\right)\right) \\
& =-\frac{4}{5} \zeta_{\phi}-\frac{4}{15} f_{\mathrm{BSM}} S_{\sigma},
\end{aligned}
$$

To obtain the last relation we have used $\zeta_{\mathrm{HS}}=\zeta_{\phi}$ and $\zeta_{\mathrm{BSM}}=\zeta_{\sigma}$ since the HS and the BSM sector respectively inherit the fluctuations of $\phi$ and $\sigma$ fields. We have also used the standard definition $S_{\sigma} \equiv 3\left(\zeta_{\sigma}-\zeta_{\phi}\right)$.

\section{A.2 Large-scale GW anisotropy}

Now we repeat the calculation for GW perturbations. Taking into account the redshift and the fact the GWs were generated during $\mathrm{RD}$ we can again write,

$$
\left.\frac{\Delta T}{T}\right|_{\mathrm{GW}}=\frac{1}{4} \delta_{\mathrm{GW}}^{\mathrm{prim}}+\Phi_{\mathrm{RD}}=\zeta_{\mathrm{GW}}-\frac{4}{3} \zeta_{\mathrm{RD}}
$$


Using the fact that $\zeta_{\mathrm{RD}}=\zeta_{\gamma}+\frac{1}{3} f_{\mathrm{GW}} S_{\mathrm{GW}}$,

$$
\left.\frac{\Delta T}{T}\right|_{\mathrm{GW}}=-\frac{1}{3} \zeta_{\mathrm{RD}}+\frac{1}{3}\left(1-f_{\mathrm{GW}}\right) S_{\mathrm{GW}} .
$$

As before, it is convenient to write the above in terms of uncorrelated perturbations,

$$
\begin{aligned}
\left.\delta_{\mathrm{GW}} \equiv 4 \frac{\Delta T}{T}\right|_{\mathrm{GW}} & =-\frac{4}{3} \zeta_{\mathrm{RD}}+\frac{4}{3}\left(1-f_{\mathrm{GW}}\right) S_{\mathrm{GW}} \\
& \approx-\frac{4}{3} \zeta_{\phi}+\frac{4}{3} S_{\sigma}\left(1-\frac{4}{3} f_{\mathrm{BSM}}\right),
\end{aligned}
$$

where to get to the last expression we have used, $f_{\mathrm{GW}} \ll 1$.

Open Access. This article is distributed under the terms of the Creative Commons Attribution License (CC-BY 4.0), which permits any use, distribution and reproduction in any medium, provided the original author(s) and source are credited.

\section{References}

[1] LIGO Scientific, Virgo collaboration, Observation of gravitational waves from a binary black hole merger, Phys. Rev. Lett. 116 (2016) 061102 [arXiv:1602.03837] [InSPIRE].

[2] LIGO ScIENTIFIC, VIRGO collaboration, GW170817: observation of gravitational waves from a binary neutron star inspiral, Phys. Rev. Lett. 119 (2017) 161101 [arXiv:1710.05832] [INSPIRE].

[3] LISA collaboration, Laser Interferometer Space Antenna, arXiv:1702.00786 [INSPIRE].

[4] KAGRA collaboration, Detector configuration of KAGRA: the Japanese cryogenic gravitational-wave detector, Class. Quant. Grav. 29 (2012) 124007 [arXiv:1111.7185] [INSPIRE].

[5] C.S. Unnikrishnan, IndIGO and LIGO-India: scope and plans for gravitational wave research and precision metrology in India, Int. J. Mod. Phys. D 22 (2013) 1341010 [arXiv: 1510.06059] [INSPIRE].

[6] M. Punturo et al., The Einstein telescope: a third-generation gravitational wave observatory, Class. Quant. Grav. 27 (2010) 194002 [INSPIRE].

[7] D. Reitze et al., Cosmic explorer: the U.S. contribution to gravitational-wave Astronomy beyond LIGO, Bull. Am. Astron. Soc. 51 (2019) 035 [arXiv:1907.04833] [InSPIRE].

[8] N. Seto, S. Kawamura and T. Nakamura, Possibility of direct measurement of the acceleration of the universe using $0.1 \mathrm{~Hz}$ band laser interferometer gravitational wave antenna in space, Phys. Rev. Lett. 87 (2001) 221103 [astro-ph/0108011] [INSPIRE].

[9] S. Kawamura et al., The Japanese space gravitational wave antenna: DECIGO, Class. Quant. Grav. 28 (2011) 094011 [InSPIRE].

[10] K.A. Kuns, H. Yu, Y. Chen and R.X. Adhikari, Astrophysics and cosmology with a decihertz gravitational-wave detector: TianGO, Phys. Rev. D 102 (2020) 043001 [arXiv:1908.06004] [INSPIRE].

[11] J. Crowder and N.J. Cornish, Beyond LISA: exploring future gravitational wave missions, Phys. Rev. D 72 (2005) 083005 [gr-qc/0506015] [INSPIRE]. 
[12] G.M. Harry, P. Fritschel, D.A. Shaddock, W. Folkner and E.S. Phinney, Laser interferometry for the big bang observer, Class. Quant. Grav. 23 (2006) 4887 [Erratum ibid. 23 (2006) 7361] [INSPIRE].

[13] M.A. McLaughlin, The North American Nanohertz Observatory for gravitational waves, Class. Quant. Grav. 30 (2013) 224008 [arXiv:1310.0758] [inSPIRE].

[14] M. Kramer and D.J. Champion, The european pulsar timing array and the large european array for pulsars, Class. Quant. Grav. 30 (2013) 224009.

[15] R.N. Manchester et al., The Parkes Pulsar Timing Array Project, Publ. Astron. Soc. Austral. 30 (2013) 17 [arXiv:1210.6130] [INSPIRE].

[16] G. Janssen et al., Gravitational wave astronomy with the SKA, PoS (AASKA14) 037 [arXiv: 1501.00127] [INSPIRE].

[17] C. Caprini and D.G. Figueroa, Cosmological backgrounds of gravitational waves, Class. Quant. Grav. 35 (2018) 163001 [arXiv:1801.04268] [INSPIRE].

[18] N. Christensen, Stochastic gravitational wave backgrounds, Rept. Prog. Phys. 82 (2019) 016903 [arXiv: 1811.08797] [INSPIRE].

[19] A. Mazumdar and G. White, Review of cosmic phase transitions: their significance and experimental signatures, Rept. Prog. Phys. 82 (2019) 076901 [arXiv:1811.01948] [InSPIRE].

[20] P. Schwaller, Gravitational waves from a dark phase transition, Phys. Rev. Lett. 115 (2015) 181101 [arXiv: 1504.07263] [INSPIRE].

[21] J. Jaeckel, V.V. Khoze and M. Spannowsky, Hearing the signal of dark sectors with gravitational wave detectors, Phys. Rev. D 94 (2016) 103519 [arXiv:1602.03901] [INSPIRE].

[22] D. Croon, V. Sanz and G. White, Model discrimination in gravitational wave spectra from dark phase transitions, JHEP 08 (2018) 203 [arXiv:1806.02332] [INSPIRE].

[23] Y. Cui, M. Lewicki, D.E. Morrissey and J.D. Wells, Probing the pre-BBN universe with gravitational waves from cosmic strings, JHEP 01 (2019) 081 [arXiv:1808.08968] [INSPIRE].

[24] M. Breitbach, J. Kopp, E. Madge, T. Opferkuch and P. Schwaller, Dark, cold, and noisy: constraining secluded hidden sectors with gravitational waves, JCAP 07 (2019) 007 [arXiv: 1811.11175] [INSPIRE].

[25] W.-C. Huang, M. Reichert, F. Sannino and Z.-W. Wang, Testing the dark SU(N) Yang-Mills theory confined landscape: From the lattice to gravitational waves, Phys. Rev. D 104 (2021) 035005 [arXiv: 2012.11614] [INSPIRE].

[26] N. Arkani-Hamed, T. Han, M. Mangano and L.-T. Wang, Physics opportunities of a $100 \mathrm{TeV}$ proton-proton collider, Phys. Rept. 652 (2016) 1 [arXiv:1511.06495] [INSPIRE].

[27] C. Cutler, Angular resolution of the LISA gravitational wave detector, Phys. Rev. D 57 (1998) 7089 [gr-qc/9703068] [INSPIRE].

[28] H. Kudoh and A. Taruya, Probing anisotropies of gravitational-wave backgrounds with a space-based interferometer: geometric properties of antenna patterns and their angular power, Phys. Rev. D 71 (2005) 024025 [gr-qc/0411017] [INSPIRE].

[29] C. Cutler and D.E. Holz, Ultra-high precision cosmology from gravitational waves, Phys. Rev. D 80 (2009) 104009 [arXiv:0906.3752] [INSPIRE].

[30] G. Cusin, C. Pitrou and J.-P. Uzan, Anisotropy of the astrophysical gravitational wave background: Analytic expression of the angular power spectrum and correlation with cosmological observations, Phys. Rev. D 96 (2017) 103019 [arXiv:1704.06184] [INSPIRE]. 
[31] G. Cusin, C. Pitrou and J.-P. Uzan, The signal of the gravitational wave background and the angular correlation of its energy density, Phys. Rev. D 97 (2018) 123527 [arXiv: 1711.11345] [INSPIRE].

[32] M. Geller, A. Hook, R. Sundrum and Y. Tsai, Primordial anisotropies in the gravitational wave background from cosmological phase transitions, Phys. Rev. Lett. 121 (2018) 201303 [arXiv: 1803.10780] [INSPIRE].

[33] N. Bartolo, V. De Luca, G. Franciolini, A. Lewis, M. Peloso and A. Riotto, Primordial black hole dark matter: LISA serendipity, Phys. Rev. Lett. 122 (2019) 211301 [arXiv:1810.12218] [INSPIRE].

[34] N. Bartolo, V. De Luca, G. Franciolini, M. Peloso, D. Racco and A. Riotto, Testing primordial black holes as dark matter with LISA, Phys. Rev. D 99 (2019) 103521 [arXiv: 1810.12224] [INSPIRE].

[35] J.D. Romano and N.J. Cornish, Detection methods for stochastic gravitational-wave backgrounds: a unified treatment, Living Rev. Rel. 20 (2017) 2 [arXiv:1608.06889] [INSPIRE].

[36] A.I. Renzini and C.R. Contaldi, Mapping incoherent gravitational wave backgrounds, Mon. Not. Roy. Astron. Soc. 481 (2018) 4650 [arXiv: 1806.11360] [InSPIRE].

[37] C.R. Contaldi et al., Maximum likelihood map-making with the Laser Interferometer Space Antenna, Phys. Rev. D 102 (2020) 043502 [arXiv:2006.03313] [INSPIRE].

[38] N. Bartolo et al., Anisotropies and non-Gaussianity of the Cosmological Gravitational Wave Background, Phys. Rev. D 100 (2019) 121501 [arXiv:1908.00527] [INSPIRE].

[39] N. Bartolo et al., Characterizing the cosmological gravitational wave background: anisotropies and non-Gaussianity, Phys. Rev. D 102 (2020) 023527 [arXiv: 1912.09433] [INSPIRE].

[40] C. Caprini et al., Science with the space-based interferometer eLISA. II: Gravitational waves from cosmological phase transitions, JCAP 04 (2016) 001 [arXiv:1512.06239] [INSPIRE].

[41] C. Caprini et al., Detecting gravitational waves from cosmological phase transitions with LISA: an update, JCAP 03 (2020) 024 [arXiv: 1910.13125] [INSPIRE].

[42] M. Hindmarsh, S.J. Huber, K. Rummukainen and D.J. Weir, Numerical simulations of acoustically generated gravitational waves at a first order phase transition, Phys. Rev. D 92 (2015) 123009 [arXiv: 1504.03291] [INSPIRE].

[43] P. Niksa, M. Schlederer and G. Sigl, Gravitational waves produced by compressible MHD turbulence from cosmological phase transitions, Class. Quant. Grav. 35 (2018) 144001 [arXiv: 1803.02271] [INSPIRE].

[44] A. Kosowsky, M.S. Turner and R. Watkins, Gravitational radiation from colliding vacuum bubbles, Phys. Rev. D 45 (1992) 4514 [InSPIRE].

[45] A. Kosowsky, M.S. Turner and R. Watkins, Gravitational waves from first order cosmological phase transitions, Phys. Rev. Lett. 69 (1992) 2026 [InSPIRE].

[46] A. Kosowsky and M.S. Turner, Gravitational radiation from colliding vacuum bubbles: envelope approximation to many bubble collisions, Phys. Rev. D 47 (1993) 4372 [astro-ph/9211004] [INSPIRE].

[47] M. Kamionkowski, A. Kosowsky and M.S. Turner, Gravitational radiation from first order phase transitions, Phys. Rev. D 49 (1994) 2837 [astro-ph/9310044] [INSPIRE].

[48] S.J. Huber and T. Konstandin, Gravitational wave production by collisions: more bubbles, JCAP 09 (2008) 022 [arXiv:0806.1828] [INSPIRE]. 
[49] R. Jinno and M. Takimoto, Gravitational waves from bubble dynamics: beyond the envelope, JCAP 01 (2019) 060 [arXiv: 1707.03111] [InSPIRE].

[50] T. Konstandin, Gravitational radiation from a bulk flow model, JCAP 03 (2018) 047 [arXiv: 1712.06869] [INSPIRE].

[51] M. Lewicki and V. Vaskonen, Gravitational wave spectra from strongly supercooled phase transitions, Eur. Phys. J. C 80 (2020) 1003 [arXiv:2007.04967] [INSPIRE].

[52] M. Lewicki and V. Vaskonen, Gravitational waves from colliding vacuum bubbles in gauge theories, Eur. Phys. J. C 81 (2021) 437 [arXiv:2012.07826] [INSPIRE].

[53] K. Schmitz, New sensitivity curves for gravitational-wave signals from cosmological phase transitions, JHEP 01 (2021) 097 [arXiv: 2002.04615] [INSPIRE].

[54] L. Randall and G. Servant, Gravitational waves from warped spacetime, JHEP 05 (2007) 054 [hep-ph/0607158] [INSPIRE].

[55] T. Konstandin and G. Servant, Cosmological consequences of nearly conformal dynamics at the TeV scale, JCAP 12 (2011) 009 [arXiv: 1104.4791] [INSPIRE].

[56] P. Baratella, A. Pomarol and F. Rompineve, The Supercooled Universe, JHEP 03 (2019) 100 [arXiv: 1812.06996] [INSPIRE].

[57] E. Megías, G. Nardini and M. Quirós, Cosmological phase transitions in warped space: gravitational waves and collider signatures, JHEP 09 (2018) 095 [arXiv:1806. 04877] [INSPIRE].

[58] K. Agashe, P. Du, M. Ekhterachian, S. Kumar and R. Sundrum, Cosmological phase transition of spontaneous confinement, JHEP 05 (2020) 086 [arXiv:1910.06238] [INSPIRE].

[59] K. Agashe, P. Du, M. Ekhterachian, S. Kumar and R. Sundrum, Phase transitions from the fifth dimension, JHEP 02 (2021) 051 [arXiv: 2010.04083] [INSPIRE].

[60] Planck collaboration, Planck 2018 results. VI. Cosmological parameters, Astron. Astrophys. 641 (2020) A6 [Erratum ibid. 652 (2021) C4] [arXiv: 1807.06209] [INSPIRE].

[61] R.K. Sachs and A.M. Wolfe, Perturbations of a cosmological model and angular variations of the microwave background, Astrophys. J. 147 (1967) 73 [Gen. Rel. Grav. 39 (2007) 1929] [INSPIRE].

[62] M.J. Rees and D.W. Sciama, Large scale density inhomogeneities in the universe, Nature 217 (1968) 511 [INSPIRE].

[63] S. Olmez, V. Mandic and X. Siemens, Anisotropies in the gravitational-wave stochastic background, JCAP 07 (2012) 009 [arXiv:1106.5555] [INSPIRE].

[64] C.R. Contaldi, Anisotropies of gravitational wave backgrounds: a line of sight approach, Phys. Lett. B 771 (2017) 9 [arXiv:1609.08168] [InSPIRE].

[65] G. Cusin, I. Dvorkin, C. Pitrou and J.-P. Uzan, First predictions of the angular power spectrum of the astrophysical gravitational wave background, Phys. Rev. Lett. 120 (2018) 231101 [arXiv: 1803.03236] [INSPIRE].

[66] A.C. Jenkins and M. Sakellariadou, Anisotropies in the stochastic gravitational-wave background: Formalism and the cosmic string case, Phys. Rev. D 98 (2018) 063509 [arXiv: 1802.06046] [INSPIRE].

[67] D. Baumann, Inflation, arXiv:0907.5424 [INSPIRE].

[68] E. Thrane and J.D. Romano, Sensitivity curves for searches for gravitational-wave backgrounds, Phys. Rev. D 88 (2013) 124032 [arXiv:1310.5300] [INSPIRE]. 
[69] V. Corbin and N.J. Cornish, Detecting the cosmic gravitational wave background with the big bang observer, Class. Quant. Grav. 23 (2006) 2435 [gr-qc/0512039] [INSPIRE].

[70] J. Baker et al., High angular resolution gravitational wave astronomy, Exper. Astron. 51 (2021) 1441 [arXiv: 1908.11410] [INSPIRE].

[71] G. Mentasti and M. Peloso, ET sensitivity to the anisotropic stochastic gravitational wave background, JCAP 03 (2021) 080 [arXiv:2010.00486] [INSPIRE].

[72] T. Regimbau, The astrophysical gravitational wave stochastic background, Res. Astron. Astrophys. 11 (2011) 369 [arXiv:1101.2762] [INSPIRE].

[73] C. Cutler and J. Harms, BBO and the neutron-star-binary subtraction problem, Phys. Rev. D 73 (2006) 042001 [gr-qc/0511092] [INSPIRE].

[74] J. Harms, C. Mahrdt, M. Otto and M. Priess, Subtraction-noise projection in gravitational-wave detector networks, Phys. Rev. D 77 (2008) 123010 [arXiv:0803.0226] [INSPIRE].

[75] K. Yagi and N. Seto, Detector configuration of DECIGO/BBO and identification of cosmological neutron-star binaries, Phys. Rev. D 83 (2011) 044011 [Erratum ibid. 95 (2017) 109901] [arXiv: 1101.3940] [INSPIRE].

[76] T. Regimbau, M. Evans, N. Christensen, E. Katsavounidis, B. Sathyaprakash and S. Vitale, Digging deeper: observing primordial gravitational waves below the binary black hole produced stochastic background, Phys. Rev. Lett. 118 (2017) 151105 [arXiv:1611.08943] [INSPIRE].

[77] S. Sachdev, T. Regimbau and B.S. Sathyaprakash, Subtracting compact binary foreground sources to reveal primordial gravitational-wave backgrounds, Phys. Rev. D 102 (2020) 024051 [arXiv : 2002.05365] [INSPIRE].

[78] A. Sharma and J. Harms, Searching for cosmological gravitational-wave backgrounds with third-generation detectors in the presence of an astrophysical foreground, Phys. Rev. D 102 (2020) 063009 [arXiv:2006.16116] [InSPIRE].

[79] M. Pieroni and E. Barausse, Foreground cleaning and template-free stochastic background extraction for LISA, JCAP 07 (2020) 021 [Erratum ibid. 09 (2020) E01] [arXiv: 2004.01135] [INSPIRE].

[80] S. Biscoveanu, C. Talbot, E. Thrane and R. Smith, Measuring the primordial gravitational-wave background in the presence of astrophysical foregrounds, Phys. Rev. Lett. 125 (2020) 241101 [arXiv:2009.04418] [INSPIRE].

[81] B.C. Barish, S. Bird and Y. Cui, Impact of a midband gravitational wave experiment on detectability of cosmological stochastic gravitational wave backgrounds, Phys. Rev. D 103 (2021) 123541 [arXiv:2012.07874] [INSPIRE].

[82] Planck collaboration, Planck 2018 results. IX. Constraints on primordial non-Gaussianity, Astron. Astrophys. 641 (2020) A9 [arXiv:1905.05697] [INSPIRE].

[83] D.H. Lyth and A. R. Liddle, The primordial density perturbation: cosmology, inflation and the origin of structure, Cambridge University Press, Cambridge U.K. (2009).

[84] K.A. Malik and D. Wands, Cosmological perturbations, Phys. Rept. 475 (2009) 1 [arXiv: 0809.4944] [INSPIRE].

[85] R.H. Cyburt, B.D. Fields, K.A. Olive and T.-H. Yeh, Big Bang nucleosynthesis: 2015, Rev. Mod. Phys. 88 (2016) 015004 [arXiv:1505.01076] [INSPIRE].

[86] P. Creminelli, On non-Gaussianities in single-field inflation, JCAP 10 (2003) 003 [astro-ph/0306122] [INSPIRE]. 
[87] D. Babich, P. Creminelli and M. Zaldarriaga, The shape of non-Gaussianities, JCAP 08 (2004) 009 [astro-ph/0405356] [INSPIRE].

[88] T. Giannantonio, C. Porciani, J. Carron, A. Amara and A. Pillepich, Constraining primordial non-Gaussianity with future galaxy surveys, Mon. Not. Roy. Astron. Soc. 422 (2012) 2854 [arXiv: 1109.0958] [inSPIRE].

[89] CMB-S4 collaboration, CMB-S4 science book, first edition, arXiv:1610.02743 [INSPIRE].

[90] C. Gordon, D. Wands, B.A. Bassett and R. Maartens, Adiabatic and entropy perturbations from inflation, Phys. Rev. D 63 (2000) 023506 [astro-ph/0009131] [INSPIRE].

[91] D. Langlois and A. Lepidi, General treatment of isocurvature perturbations and non-Gaussianities, JCAP 01 (2011) 008 [arXiv: 1007.5498] [InSPIRE].

[92] J. Fonseca and D. Wands, Primordial non-Gaussianity from mixed inflaton-curvaton perturbations, JCAP 06 (2012) 028 [arXiv: 1204.3443] [INSPIRE].

[93] J.E. Kim, H.P. Nilles and M. Peloso, Completing natural inflation, JCAP 01 (2005) 005 [hep-ph/0409138] [INSPIRE].

[94] S. Kumar and R. Sundrum, Cosmological collider physics and the curvaton, JHEP 04 (2020) 077 [arXiv: 1908.11378] [INSPIRE]. 